\title{
Functional Outcome of Fractures Distal End of Femur Using LCP - A Prospective Observational Study
}

\author{
Shikhar Yadav ${ }^{a}$, Jithin Mohan ${ }^{a}$, Dileep S ${ }^{a}$, Divya Ga, Joice Varghese ${ }^{a}$, John T John ${ }^{a}$ \\ a. Department of Orthopaedics, Lourdes Hospital, Ernakulam, Kerala
}

Published on $1^{\text {st }}$ June 2020

\begin{abstract}
Background: Trauma management is an ever evolving part of orthopaedics and with the latest advancement in metallurgy, crafting processes and biomechanical studies, the implants used have been continuously changing. Distal femur fractures occur at a major joint and their management has always been complicated ranging from preoperative, intra-op to postoperative period. All the studies and research are aimed towards achieving the best functional outcome and functional outcome studies help in collecting and assimilation of the data for the same which further help in modification of techniques to further improve the same. This study focusses on the outcomes achieved by using the distal femur locking compression plate for the management of fractures distal end of femur.
\end{abstract}

Materials and Methods: Prospective, unicentric, observational study from June 2018 - February 2020. All patients with distal femur facture were included. Pathological fractures, open fractures, fractures older than 3 weeks and patients with ipsilateral proximal tibia, patella and proximal femur were excluded. Surgical approach was surgeon's choice. Functional outcome was measured using Knee Society Knee Scoring system at 6weeks, 3 months, 6months and 12 months. Functional outcome measured at 12 months was used for evaluation.

Results: 28 patients, 19(67.9\%) males and 9(32.1\%) females. 89.47\% of males sustained the fracture due to road traffic accident and $66.66 \%$ of females sustained due to fall and all belonged to age group more than 60 years. The average range of motion achieved was $112.3 \pm 8.2$. Functional outcome at 12 months $71.4 \%$ patients had excellent, $25 \%$ had good and $3.6 \%$ patients had fair and no patient had poor outcome.

Conclusion: Distal femur fracture is more common in the age group of 21-40 years. The average range of motion of knee achieved in Type A and Type B fractures are significantly better than Type $C$ fractures. Excellent functional outcome was statistically significant in Type A and Type B fractures when compared to Type $C$ fractures.

Key Words: Fracture distal end of femur, Distal femur locking compression plate, Functional outcome, Knee Society Knee Score, Prospective unicentric observational study

\section{INTRODUCTION}

$\mathrm{K}$ nee joint is one of the most important joint required for mobilization and distal femur is one of the most critical load bearing areas in the human body. Distal femur fractures account for 4-7\% of all femur fractures which approximates to $37 / 100,000$ person years. ${ }^{1}$ The rapid industrialization has resulted in the increase in the number of road traffic accidents affecting many young lives. The bumper of the car reaches the level of the knee and often results in distal femur fractures. ${ }^{2}$

An axial load along with a varus, valgus or rotational forces results in a fracture of the distal femur. ${ }^{3}$ High energy trauma and osteoporosis often results in comminuted intraarticular fractures and intraarticular fractures of the distal femur present a huge surgical challenge. ${ }^{4}$ Distal femur fracture causes malalignment and instability and restriction of motion around the knee joint. Classification of these fractures act as an important guide for the management of

Cite this article as: Yadav S, Mohan J, S D, G D, Varghese J, John JT. Functional Outcome of Fractures Distal End of Femur Using LCP - A Prospective Observational Study. KJOrth. 2020 Jun 1;33(01):13-7. 


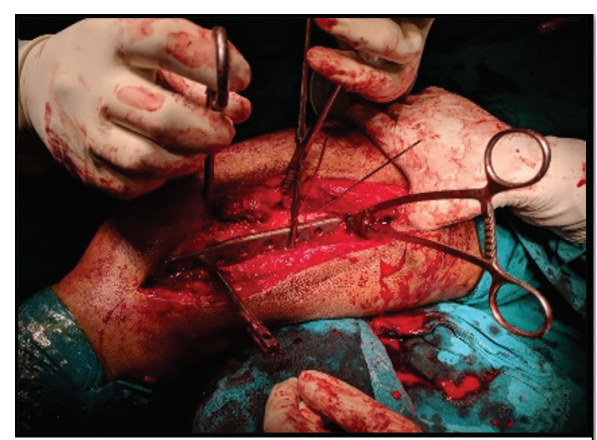

Figure 1. Lateral approach for distal femur fracture

treatment. Most of the fractures heal readily with protected weight bearing. The fate of a joint after a fracture depends on the post traumatic arthrosis that develops. A healthy articular surface is necessary for a stable painless free joint and the guidelines for management of intraarticular fractures include: 1. Anatomical reduction 2. Absolute stability 3 . Early mobilization. ${ }^{5}$

Despite the advances, operating distal femur fracture remain a technical challenge. High velocity trauma often results in severely comminuted fractures. The comminution creates a challenge for the reconstruction of articular surface.

\section{MATERIALS AND METHODS}

It was a prospective, unicentric, observational study from the period January 2018 to February 2020. By taking $p$ as $0.037 \%{ }^{5}$ with a confidence of $95 \%$, an error of estimate of $8 \%$, the minimum sample for the study as per the above formula worked out to be 23. Patients less than 18 years of age, fractures older than 3 weeks, open fractures, pathological fractures, patients with neurovascular deficit and other fractures involving ipsilateral proximal femur, patella and proximal tibia were excluded from the study. SPSS version 20 was used for statistical analysis. Chi-square test/ Fischer's exact test was performed to find the relationship between functional outcome and other variable. A p-value less than 0.05 was taken as statistically significant.

Patients presented to the casualty with history of trauma. They were managed initially according to Advanced

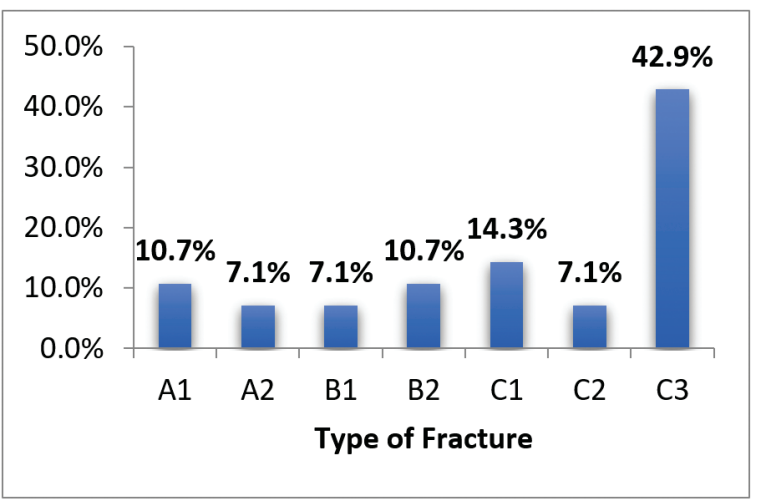

Figure 2. Distribution of type of fracture
Trauma Life Support (ATLS) protocol. Suspected fractures were immobilized using splints. Patient was informed regarding the study and consent taken. All cases were operated by the same trauma surgeon having more than 10 years of surgical experience. Surgical approach was based on the fracture pattern and the need for the access to the articular surface. Anatomical reduction of the articular surface and acceptable reduction of the metaphyseal fractures were of prime importance and surgical approach was decided to achieve them. The approaches used were: 1. Lateral approach (Figure 1) 2. Minimally Invasive plate osteosynthesis and 3. The swashbuckler approach.

Postoperatively all patients were given a knee brace and physiotherapy with knee ROM exercises, ankle pump exercises, static quadriceps exercises for quadriceps along with straight leg raising exercises aided by a knee brace were started from Day 1. Non weight bearing walking with the help of a walker was started on post op day 1 . At 6 weeks radiological union was assessed. At 3 months full weight bearing was started in patients showing adequate radiological union. Patients were followed up for at least 12 months.

Postoperatively radiographs were assessed at 6 weeks, 12 weeks, 6 months and 12 months. Postoperative outcomes were measured by calculating Knee Society Knee Scoring system $(\mathrm{KSS})^{6}$ at 6 weeks, 12 weeks, 6 months, and 12 months interval after surgery. The scoring system has both objective and functional scores. Functional score obtained at 12 months was used for statistical analysis (Figures $\mathbf{4} \& \mathbf{5}$ )

\section{RESULTS}

A total of 28 patients participated in the study which included 19(67.9\%) males and 9(32.1\%) females with a mean age of $48.53( \pm 17.6) .39 .3 \%$ patients fell under the age group of 21-40 years, $28.6 \%$ under the age group of 41-60 and $32.1 \%$ of patients were $>60$ years in age. Mechanism of injury was found to be due to road traffic accident in $71.4 \%$ patients and due to a fall in $28.6 \%$ patients.

On further evaluating the distribution of age, sex and mechanism, it was found than amongst 19 male cases,

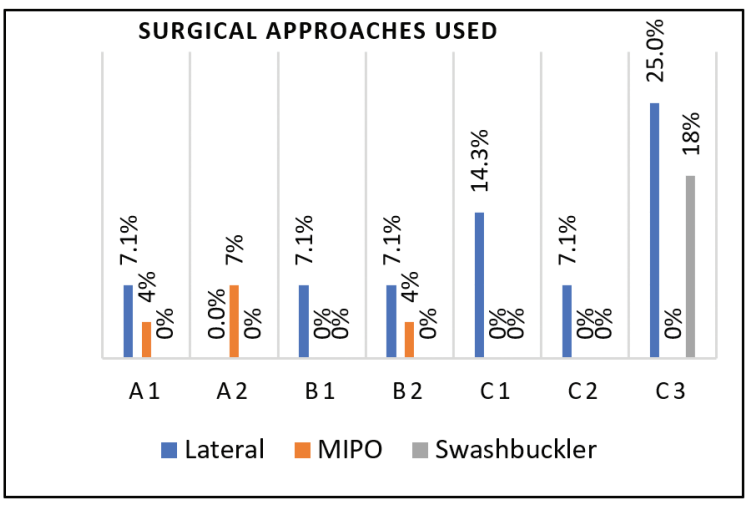

Figure 3. Distribution of use of surgical approach 

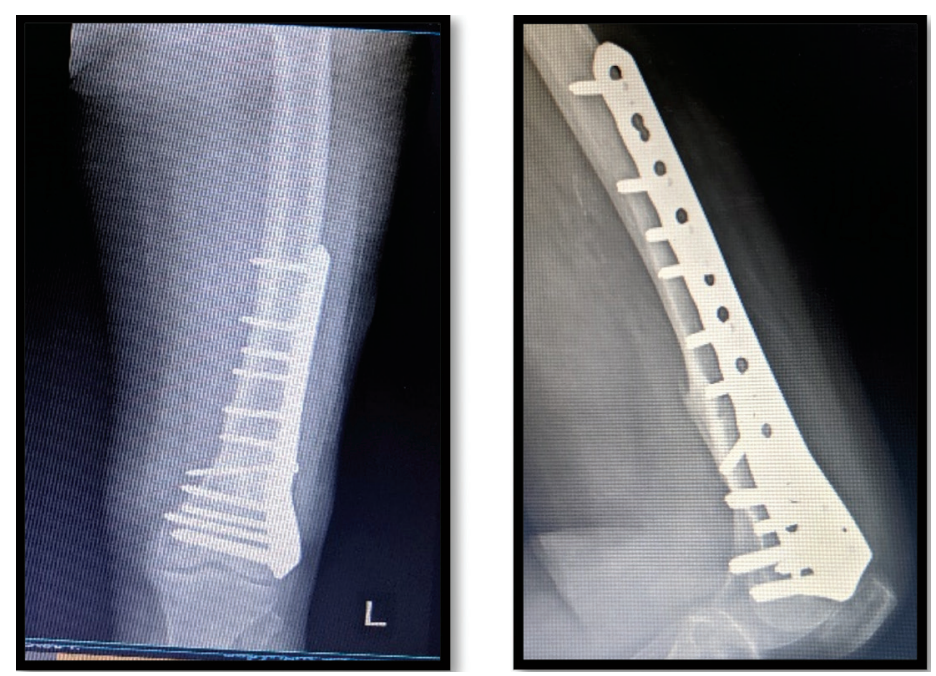

Figure 4. Xray of left femur AP and lateral view at 12 months

17(89.47\%) were due RTA and $2(10.52) \%$ were due to fall. Amongst the 9 female cases, 3(33.33\%) were due to RTA and 6(66.66\%) were due to fall. Amongst 11 cases in age group of $21-40$ years, all $11(100 \%)$ were due to RTA and none due to fall and amongst the 9 cases with age more than 60 years, $1(11.11 \%)$ had RTA while the other $8(88.88 \%)$ were due to a fall out of which $6(75 \%)$ were females. This indicated the bimodal distribution of fractures with high energy fractures occurring due to RTA in young males and low energy fractures occurring in older females due to osteoporosis.

Functional outcome obtained using the Knee Society Knee Scoring system was excellent in 20(71.4\%) patients, good in $7(25 \%)$ cases and fair in $1(3.6 \%)$ case at the end of one year. No case of poor functional outcome was seen in the study. Relationship between functional outcome and age, functional outcome and sex and functional outcome and mechanism of injury were statistically insignificant with $\mathrm{p}$ - values $0.875,0.214$ and 0.371 respectively. Functional outcome showed a continuous increase from 3 months to 1 year. At 3 months, it was $31.25 \pm 11.23$, at 6 months it increased to $66.25 \pm 10.23$ to $80 \pm 9.25$ at 1 year. Functional outcome obtained in Type $\mathrm{C}$ fracture was significantly lower when compared to Type A and Type B fractures combined with a p-value of 0.049 . The average range of flexion of knee achieved in the study was $112.3^{\circ} \pm 8.2^{\circ}$.

Superficial infection was seen in $7.1 \%$ of patients. In 3 patients, full weight bearing was delayed till 4 months due to insufficient callus formation over medial cortex. No cases of deep infection, non-union or implant failure were seen. To prevent varus collapse a medial plate was used in one Type A2 and one Type C2 and one Type C3 fractures. Cannulated cancellous screws were used in 7 Type C fractures (Figures $\mathbf{2} \& \mathbf{3}$ ).

\section{DISCUSSION}

Singhal et al. ${ }^{7}$ did a study on functional outcome of distal femur fractures using distal femur locking compression

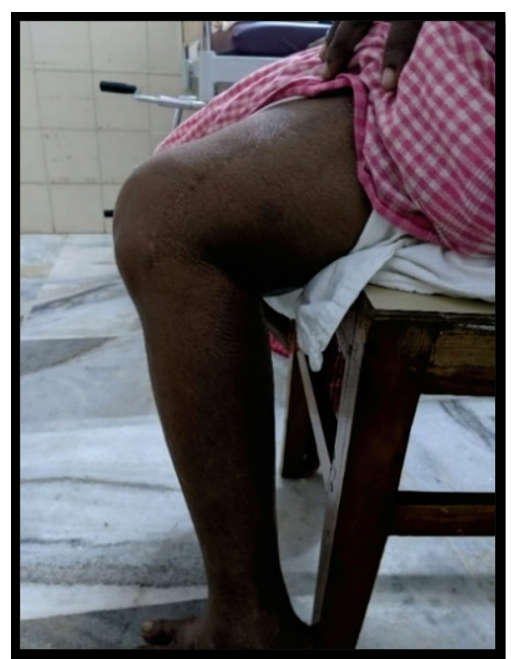

Figure 5. Flexion of left knee at 12 months post OP

plate and the mean age of patients was 38.06 years. Yeap et al. ${ }^{8}$ and Virk et al. ${ }^{9}$ did a similar study and had a mean age of 44 years and 36.64 years. In the present study mean age was 48.54 years. It was seen that $11(39.3 \%)$ of patients were young patients less than 40 years and $9(32.1 \%)$ more than 60 years formed two major groups of study population. On further analysis it was seen that of the 11 patients in the age group 21-40 10(90.9\%) of the patients were male and of the 9 patients in the age group above 60 years, $6(66.6 \%)$ of the patients were male which may be attributed to the increased number of road traffic accidents seen amongst young men and increased osteoporosis in elderly women.

Konuganti et al. ${ }^{10}$ in their study had $80 \%$ male and $20 \%$ female participants which is similar to the study conducted by Machi et al. ${ }^{11}$ having $70 \%$ of males and $30 \%$ female participants. Fankhauser et al. ${ }^{12}$ in his study had an almost equal preponderance with $55.2 \%$ males and $44.8 \%$ females. In our study, male preponderance was seen with $19(67.9 \%)$ of patients being males and $17(32.1 \%)$ females. Of the $19(67.9 \%)$ male cases in the study, $17(89.5 \%)$ cases sustained the fracture due to road traffic accidents and amongst the $9(32.1 \%)$ female cases $3(33.34 \%)$ were due to road traffic accidents and 6(66.6\%) were due to fall and all of them were above the age of 60 years.

Yeap et al. ${ }^{8}$ in his retrospective study of patients with distal femur fractures found $63.6 \%$ of patients to have sustained the fracture due to a road traffic accident and $36.4 \%$ of patients to have sustained it due to a fall. Road traffic accidents were found to be a major cause of the fracture in a study conducted by Koniganti et al. ${ }^{10}$ and Padha et al. ${ }^{13}$ comprising of $55.5 \%$ and $80 \%$ of fractures as compared to $45.5 \%$ and $20 \%$ of fractures caused by a fall in the respective studies. Road traffic accidents were found to be the major cause in our study as well comprising $71.4 \%$ of patients whereas only $28.6 \%$ of fractures occurred due to a fall. Road traffic accidents are high velocity injuries and were seen most amongst the young male population. 


\begin{tabular}{|c|c|c|c|c|}
\hline Paramerers & N & Mean & Min & Max \\
\hline $\mathrm{A} 1$ & $10.7 \%$ & \multirow{3}{*}{$22 \%$} & $9.9 \%$ & \multirow{3}{*}{$34.5 \%$} \\
\hline A2 & $7.1 \%$ & & $16 \%$ & \\
\hline A3 & - & & $62 \%$ & \\
\hline B1 & $7.1 \%$ & \multirow{2}{*}{$16 \%$} & - & - \\
\hline B2 & $10.7 \%$ & & $11.35 \%$ & - \\
\hline $\mathrm{C} 1$ & $14.3 \%$ & \multirow{3}{*}{$62 \%$} & $11.71 \%$ & \\
\hline $\mathrm{C} 2$ & $7.1 \%$ & & $18.72 \%$ & $31 \%$ \\
\hline C3 & $42.9 \%$ & & $4.47 \%$ & $34.5 \%$ \\
\hline
\end{tabular}

\begin{tabular}{ccc}
\multicolumn{3}{c}{ Table 2. Comparison of average range of motion achieved } \\
\hline Sl.no & Study & Average knee ROM \\
\hline 1. & ${\text { Fankhauser et al. } .^{12}}^{\circ}$ & $113^{\circ}$ \\
\hline 2. & Mark Weight et al. $^{14}$ & $114^{\circ}$ \\
\hline 3. & Kayali et al. $^{15}$ & $117.3^{\circ}$ \\
\hline 4. & Markmiller M et al. ${ }^{16}$ & $110^{\circ}$ \\
\hline 5. & Present study & $112^{\circ}$ \\
\hline
\end{tabular}

In a prospective study conducted by Padha et al. 13 and Fankhauser et al., ${ }^{12}$ they had majority of the cases of Type $\mathrm{C}$ fractures and very few cases of type B fractures as seen in the table. Koniganti et al. ${ }^{10}$ on the other hand had majority of Type A fractures.

In this study we had majority of Type $C$ fractures and an equal distribution of Type B and type A fractures. More preponderance of Type $\mathrm{C}$ fractures maybe contributed to majority of the fractures occurring due to road traffic accident which are high velocity injuries.

Average range of knee flexion achieved by Fankhauser et al., ${ }^{12}$ Mark Weight et al., ${ }^{14}$ Kayali et al. ${ }^{15}$ Markmiller M et al. ${ }^{16}$ after fixation of distal femur fractures with distal femur locking compression plate were comparable.

In our study we achieved and average range of flexion of knee of $112^{\circ}$ which is comparable to other studies. Around $28.6 \%$ of patients achieved knee flexion more than $120^{\circ}$ and $42.9 \%$ patients had knee flexion between 110 and $119^{\circ}$. In the study it was found that patients with Type A and Type B fractures achieved better range of motion of knee at one year follow up which was statistically significant with a p-value of 0.000 .

\begin{tabular}{ccccccc}
\hline \multicolumn{6}{l}{ Table 3. Comparison of functional outcome } \\
$\begin{array}{c}\text { Sl. } \\
\text { No }\end{array}$ & Study & Poor & Fair & Good & Excellent & Score \\
\hline 1. & Agrawal A et al. ${ }^{17}$ & 0 & $16.6 \%$ & $25 \%$ & $58.3 \%$ & KSS \\
\hline 2. & ${\text { Doshi et a }{ }^{18}}^{18}$ & $16.7 \%$ & $4.2 \%$ & $29.1 \%$ & $50 \%$ & KSS \\
\hline 4. & Lupescu et al. $^{19}$ & $5.55 \%$ & $8.33 \%$ & $16.6 \%$ & $70 \%$ & KSS \\
\hline 5. & Present study & 0 & $3.6 \%$ & $25 \%$ & $71.4 \%$ & KSS \\
\hline
\end{tabular}

In a prospective study conducted by Agrawal A et al., ${ }^{17}$ 12 patients with distal femur fractures were operated with lateral locked compression plate and observed. Patients had a mean follow up of 17.6 months and $58.3 \%$ patients had excellent outcome, $25 \%$ patients had fair outcome and $16.6 \%$ had fair outcome at one year follow up using knee society knee scoring system .

In a retrospective study conducted by Doshi et al, ${ }^{18} 24$ patients with distal femur fractures were treated using minimally invasive plate osteosynthesis technique were observed for a mean period of 15.3 months. They found that the mean range of motion at 6 months was $100^{\circ}$ and functional knee score using knee society knee score was 88.8. They found $50 \%$ of patients to have excellent results, $29.1 \%$ of patients with good results, $4.2 \%$ patients with fair outcome but $16.7 \%$ of patients with poor outcome.

In our study we had $71.4 \%$ of patients with excellent outcome, $25 \%$ patients with good and $3.6 \%$ patients with fair outcome. The increased percentage of excellent outcomes in our study may be attributed to the exclusion of open fractures from the study. Open fractures are associated with multiple complications and multiple soft tissue injuries. Open fractures are managed as staged procedures after wound healing resulting delay in time interval between trauma and operative management. Lupescu et al. ${ }^{19}$ did a retrospective study of 36 patients with closed distal femur fractures. Type B fractures were excluded from the study and Type A fractures were managed with retrograde intramedullary nail and Type C was managed with distal femur locking compression plate and they got excellent results ins $70 \%$ of their patient, good results in $16.6 \%$ patients, fair results in $8.33 \%$ patients and poor results in $5.55 \%$ of their patients (Tables $1,2 \mathrm{\&} 3$ ).

\section{CONCLUSION}

This study concludes that distal femur locking compression plate is an excellent implant for fixation of distal femur fractures. However anatomical reduction and early mobilization of the joint should be given the prime importance. Augmentation of the construct with a medial plate over the distal femur is encouraged especially in fractures with comminution of medial cortex in order to avoid a varus collapse. Post operative management plays an important role in order to prevent complications such as non-union, varus collapse and implant failure. This study was limited by its small sample size and 1 year follow up. Many cases of implant failure have been reported at beyond one year of follow up. Maximum range of motion of knee addressed by the Knee Society Knee Scoring system is $125^{\circ}$ and a range beyond that could not be differentiated. And a comparative study of the functional outcome using different available implants would give a better insight in the improved functional outcome by a selected implant. 


\section{Clinical Relevance}

This study adds on to the database of the functional outcome obtained in fractures distal end of femur managed with locking compression plate. This data can be used to compare upcoming modification in patient care to see whether the modification is comparatively better or not as to the outgoing treatment. Since open fractures, pathological fractures and other fractures involving the same joint have been excluded, this study provides a data not influenced by other confounding factors.

\section{END NOTE}

\section{Author Information}

1. Dr Shikhar Yadav, MBBS, DNB Resident, Department of Orthopaedics, Lourdes Hospital, Ernakulam, Kerala

2. Dr Jithin Mohan, MBBS, D. Ortho, DNB Resident, Department of Orthopaedics, Lourdes Hospital, Ernakulam, Kerala

3. Dr Dileep S, MBBS, D. Ortho, DNB (Ortho), Fellowship in Arthroplasty and Arthroscopy, Junior Consultant, Department of Orthopaedics, Lourdes Hospital, Ernakulam, Kerala

4. Dr Divya G, MBBS, DNB(Ortho), Junior Consultant, Department of Orthopaedics, Lourdes Hospital, Ernakulam, Kerala

5. Dr Joice Varghese, MBBS, D. Ortho, DNB(Ortho), Senior Consultant, Department of Orthopaedics, Lourdes Hospital, Ernakulam, Kerala

6. Dr John T John, MBBS, MS Ortho, MCh ortho(UK), MRCS(Glas.), FRCS (Glas.), FRSH (London), Dip TB (Singapore), F.NUH (Singapore). Head of Department, Department of Orthopaedics, Lourdes Hospital, Ernakulam, Kerala

\section{Conflict of Interest: None declared}

\section{REFERENCES}

1. Martinet O, Cordey J, Harder Y, Maier A, Bühler M, Barraud GE. The epidemiology of fractures of the distal femur. Injury. 2000 Sep;31 Suppl 3:C62-63.

2. Patil PB, Patil RS, Lad SA, Suren T, Bothara NV, et al. The study of functional outcome of distal end femur fractures operated with locking compression plate. Asian Pac J Health Sci. 2016 Jan;3(1):135-9.

3. Court-Brown CM, Heckman JD, McQueen MM, Ricci WM, Tornetta P, McKee MD. Rockwood and Green's fractures in adults. 2015 .
4. Kiran Kumar GN, Sharma G, Farooque K, Sharma V, Ratan R, Yadav S, et al. Locking Compression Plate in Distal Femoral Intra-Articular Fractures: Our Experience. Int Sch Res Notices. 2014;2014:372916.

5. Rüedi TP, Murphy WM. AO principles of fracture management. Stuttgart; New York; Davos Platz [Switzerland: Thieme ; AO Pub.; 2000. 864 p.

6. Insall JN, Dorr LD, Scott RD, Scott WN. Rationale of the Knee Society clinical rating system. Clin Orthop Relat Res. 1989 Nov;(248):13-4.

7. Singhal V, Gohiya A. A PROSPECTIVE STUDY ON THE OUTCOMES FOLLOWING LCP CONDYLAR PLATE FIXATION IN AO TYPE C DISTAL FEMORAL FRACTURES. Journal of Evolution of Medical and Dental Sciences. 2015 Nov 18;4:15857-62.

8. Yeap ewe juan, Deepak A. Distal Femoral Locking Compression Plate Fixation in Distal Femoral Fractures: Early Results. Malaysian Orthopaedic Journal. 2007 May 1;1:12-7.

9. Virk JS, Garg SK, Gupta P, Jangira V, Singh J, Rana S. Distal Femur Locking Plate: The Answer to All Distal Femoral Fractures. J Clin Diagn Res. 2016 Oct;10(10):RC01-5.

10. Konuganti SR, Jakinapally SR, Rao VP, Rapur S. Management of distal femur fractures with locking compression plate: a prospective study. International Journal of Research in Orthopaedics. 2018 Feb 23;4(2):208-13.

11. Machhi DR, Namsha DB, Dindod DV. Outcome of intra articular distal femur fracture treated with locking compression plate. Int $\mathrm{J}$ Orthop Sci. 2017;3(3):503-7.

12. Fankhauser F, Gruber G, Schippinger G, Boldin C, Hofer HP, Grechenig W, et al. Minimal-invasive treatment of distal femoral fractures with the LISS (Less Invasive Stabilization System): a prospective study of 30 fractures with a follow up of 20 months. Acta Orthop Scand. 2004 Feb;75(1):56-60.

13. Padha K, Singh S, Ghani A, Dang H. Distal Femur Fractures and its Treatment with Distal Femur Locking Plate. 2016;18(2):5.

14. Weight M, Collinge C. Early results of the less invasive stabilization system for mechanically unstable fractures of the distal femur (AO/OTA types A2, A3, C2, and C3). J Orthop Trauma. 2004 Sep;18(8):503-8.

15. Kayali C, Agus H, Turgut A. Successful results of minimally invasive surgery for comminuted supracondylar femoral fractures with LISS: comparative study of multiply injured and isolated femoral fractures. J Orthop Sci. 2007 Sep;12(5):458-65.

16. Markmiller M, Konrad G, Südkamp N. Femur-LISS and distal femoral nail for fixation of distal femoral fractures: are there differences in outcome and complications? Clin Orthop. 2004 Sep;(426):252-7.

17. Agrawal A, Kiyawat V. Complex AO type C3 distal femur fractures: Results after fixation with a lateral locked plate using modified swashbuckler approach. Indian J Orthop. 2017 Feb;51(1):18-27.

18. Doshi HK, Wenxian P, Burgula MV, Murphy DP. Clinical outcomes of distal femoral fractures in the geriatric population using locking plates with a minimally invasive approach. Geriatr Orthop Surg Rehabil. 2013 Mar;4(1):16-20.

19. Lupescu O, Nagea M, Patru C, Vasilache C, Popescu GI. Treatment Options for Distal Femoral Fractures. Maedica (Bucur). 2015 Jun;10(2):117-22. 